METAL REGULATION

Iron clad delivery

Cell Metab. 19, 445-457 (2014)

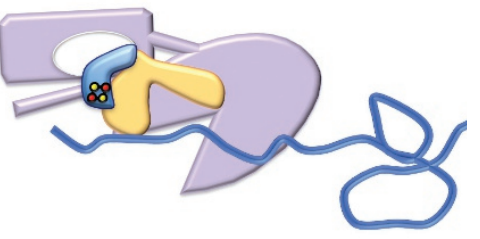

The delivery of assembled Fe-S clusters onto mitochondrial proteins is performed by a protein escort complex consisting of a chaperone (HSPA9), co-chaperone (HSC20) and the scaffold ISCU. However, it was unclear how this escort complex recognized protein targets. Maio et al. performed a yeast two-hybrid screen to identify binding partners of the co-chaperone, HSC20. They found that succinate dehydrogenase subunit $\mathrm{B}$ (SDHB), an Fe-S-containing electron transport chain complex II protein, interacted with HSC20 as part of an intermediate complex (designated as CIIb), which predated the fully assembled complex II. The incorporation of Fe-S clusters onto SDHB was thought to be a prerequisite for complex II maturation. Consistent with this, knockdown of HSC20 or HSPA9 blocked SDHB incorporation into complex II, resulting in a loss of complex II formation and activity. Deletion analysis of SDHB revealed two unique $\mathrm{L}(\mathrm{I}) \mathrm{YR}$ motifs that mediate binding to the C terminal of HSC20. Although these motifs were not sufficient for HSC20 binding, the N-terminal L(I)YR motif was critical for linking SDHB to the Fe-S-containing complex and ensuring
SDHB incorporation into complex II. Interestingly, additional HSC20 binding proteins found in electron transport chain complex I and III have L(I)YR motifs, suggesting that this recognition motif may be a generalizable mechanism to ensure proper Fe-S delivery.

\section{PLANT INNATE IMMUNITY}

\section{Tussling over tyrosine}

Science doi:10.1126/science.1248849

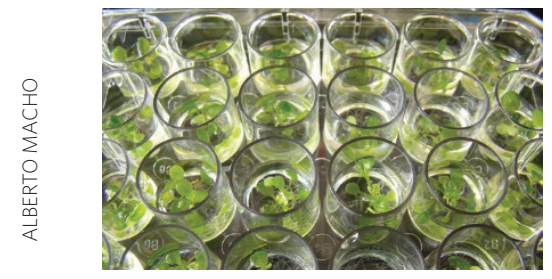

PAMP-triggered immunity (PTI) is initiated in plants when pathogen-associated molecular patterns (PAMPs) - typically fragments of pathogen biomoleculesinteract with cell-surface pattern-recognition receptors (PRRs). For instance, the EF-TU RECEPTOR (EFR) in Arabidopsis thaliana is involved in antibacterial responses initiated by elf18, a peptide fragment of bacterial elongation factor Tu. Pathogenic bacteria have also evolved countermeasures to suppress these PTI responses. Macho et al. now highlight the importance of tyrosine phosphorylation of EFR (and other non-arginine-aspartate kinases) in PTI and in the evasive actions of pathogenic bacteria. Treatment of $A$. thaliana seedlings with different tyrosine kinase inhibitors diminishes the plant's response to PAMPs. Probing or immunoprecipitating EFR

IMMUNOLOGY

\section{Interfering with Interferon}

J. Immunol. 192, 3383-3389 (2014)

Systemic lupus erythematosus (SLE) is an autoimmune disease where self single-stranded RNA (ssRNA) and double-stranded DNA (dsDNA) are recognized by innate immune system toll-like receptors (TLRs) TLR7 and TLR9 on plasmacytoid dendritic cells (pDCs), leading to inflammation and production of cytokines such as the type I interferon IFN- $\alpha$. Building on their previous finding linking the endosomal PI(3)P kinase PIKfyve in TLRdependent production of cytokines IL-12 and IL-23, Cai et al. explored potential roles for PIKfyve in the SLE system. The PIKfyve inhibitor apilimod blocked TLR7- and TLR9induced expression of type I interferons, including IFN- $\alpha$ in mouse and human pDCs. A mouse model of dysregulated PIKfyve confirmed a role for PIKfyve in TLR-induced IFN- $\alpha$ production. Gene expression analysis showed that apilimod upregulated several genes that had been stimulated by the TLR7 agonist R848, including the transcription factor ATF3, which had already been shown to be a transcriptional repressor for TLR-mediated induction of the cytokine IL-12p40. Chromatin immunoprecipitation experiments support a model where PIKfyve silencing enhances TLR-induced ATF3 expression to promote its binding and silencing of IFN genes. These results uncover a new role for PIKfyve in regulating TLR-induced type I interferons and suggest a potential new approach to SLE treatment. with anti-phosphotyrosine antibodies revealed that EFR is activated by tyrosine phosphorylation in vitro and in planta in an elf18-dependent way. Systematic mutagenesis of EFR tyrosines and in planta analysis revealed Tyr836 as a key phosphorylation site and regulator of EFR signaling activity. A targeted yeast twohybrid screen revealed that HopA01-a virulence factor from Pseudomonas syringae that has protein tyrosine phosphatase (PTP) activity - binds EFR and reduces its tyrosine phosphorylation levels in plants. Taken together, the study suggests that the phosphorylation states of key tyrosine residues in PRRs are central to PTI signaling and thus may be susceptible to counterattack by effectors that are secreted by pathogens to blunt the host immune response.

TLS

PEPTIDES

\section{A synthetic step change}

ChemBioChem doi:10.1002/cbic.201300796 ChemBioChem doi:10.1002/cbic.201300797

Synthetic peptides are important research tools across scientific fields, but obtaining these materials can be time consuming, as coupling of each amino acid is typically conducted for an hour or more. Two papers now provide a serious shake-up of these reaction conditions. Simon et al. start by revisiting the basic kinetics of each step, confirming via inline UV detection of a released FMOC protecting group that reactions are complete in seconds. Unsurprisingly, these rates are faster at elevated temperatures, but heating stored amino acid solutions can lead to degradation. The authors therefore constructed reaction vessels that use flow-based coupling, deprotection and washing steps to decrease reaction times, incorporating a heat exchanger to rapidly heat materials immediately prior to and during synthesis. The final optimized protocol takes less than two minutes to add each amino acid, with purities of diverse peptides in line with material made by standard practices. Mong et al. leverage this technology to generate peptide fragments of two model proteins, DAPRin pE59 and barnase, that are then coupled via native chemical ligation. Because the synthesis is so fast, the authors were able to identify and resolve numerous pitfalls typical of polypeptide synthesis, such as deletion products, premature chain termination and aspartimide formation, and provide guidelines for handling these problems. This new methodology should dramatically increase the availability of peptides and proteins for basic and applied studies. 